\title{
Fatty and lean red meat consumption in China: differential association with Chinese abdominal obesity
}

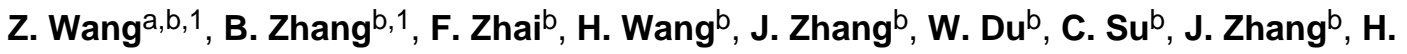 \\ Jiang $^{\mathrm{b}}$, and B. M. Popkin ${ }^{\mathrm{a},{ }^{*}}$ \\ aDepartment of Nutrition and Carolina Population Center, University of North Carolina at Chapel \\ Hill, USA \\ bNational Institute for Nutrition and Food Safety, Chinese Center for Disease Control and \\ Prevention, China
}

\section{Abstract}

\begin{abstract}
Aim-We examined the longitudinal association between red meat (RM) consumption and the risk of abdominal obesity in Chinese adults.

Methods and results-Our data are from 16,822 adults aged 18 to 75 in the China Health and Nutrition Survey from 1993 to 2011. We assessed RM intake with three 24-hour dietary recalls. We defined abdominal obesity as a waist circumference (WC) $\geq 85$ centimeters (cm) for men and $\geq 80 \mathrm{~cm}$ for women. Multilevel mixed-effect regression models showed that men experienced WC increases of $0.74 \mathrm{~cm}$ (95\% confidence interval [CI]: 0.39-1.09) from a higher total intake of fresh RM and $0.59 \mathrm{~cm}$ (95\% CI: 0.24-0. 95) from a higher intake of fatty fresh RM but $0.14 \mathrm{~cm}$ (95\% CI: -0.39-0.66) from a higher intake of lean fresh RM in the top versus the bottom quartile when adjusted for potential confounders. In contrast, after additional adjustment for baseline WC, the odds ratios of abdominal obesity in men were attenuated for total fresh RM (1.25 [95\% CI: 1.061.47]) and fatty fresh RM (1.22 [95\% CI: 1.03-1.44]) but were still not affected by lean fresh RM (0.95 [95\% CI: $0.75-1.22])$. Women also showed a positive association of fatty fresh RM intake with abdominal obesity.
\end{abstract}

(C) 2014 Elsevier B.V. All rights reserved.

"Corresponding author: Barry M. Popkin, W. R. Kenan Jr. Distinguished Professor, Carolina Population Center, University of North Carolina at Chapel Hill, CB \#8120 University Square, 123 West Franklin Street, Chapel Hill, NC 27516-3997, Phone: 919-966-1732, Fax: 919-966-9159 (backup: 919-966-6638), popkin@unc.edu.

${ }^{1}$ Equally contributed to the paper

Authors' Contributions

$\mathrm{ZW}$ contributed to the formulation of the research question, data analysis, data interpretation, and manuscript writing. BZ contributed to the data analysis, data interpretation, and manuscript writing. FZ, HW, JZ, WD, CS, JZ, and HJ contributed to the discussion and data interpretation. BMP contributed to the discussion, data interpretation, and manuscript writing. All authors read and approved the final version of the manuscript. We also thank Jean Kaplan for assistance with editing the manuscript.

Disclosure Statement

The authors declare no conflict interest.

Publisher's Disclaimer: This is a PDF file of an unedited manuscript that has been accepted for publication. As a service to our customers we are providing this early version of the manuscript. The manuscript will undergo copyediting, typesetting, and review of the resulting proof before it is published in its final citable form. Please note that during the production process errors may be discovered which could affect the content, and all legal disclaimers that apply to the journal pertain. 
Conclusion-Greater intake of fatty fresh RM was significantly associated with higher WC (men only) and abdominal obesity risk in Chinese adults. The gender-specific differential association of fatty versus lean fresh RM warrants further study.

\section{Keywords}

fatty fresh red meat; abdominal obesity; waist circumference; Chinese

\section{INTRODUCTION}

Over the past two decades, along with rapid economic growth and social changes, China has experienced marked shifts in diet and physical activity and concurrent shifts in disease patterns. ${ }^{1-3}$ Several studies have suggested that cardiometabolic risk is pervasive across rural and urban China ${ }^{4-6}$ and that abdominal obesity is highly predictive of metabolic risk in Chinese adults, irrespective of being overweight. ${ }^{7,8}$ This emphasizes the key role of waist circumference (WC) in the prevention of cardiovascular disease, the leading cause of mortality in China. Study results show a rapid increase in abdominal obesity among Chinese adults between 1993 and 2006 from $17.9 \%$ to $42.5 \%$ for men and from $28.8 \%$ to $46.9 \%$ for women. ${ }^{9}$ It is therefore important to identify modifiable risk factors, such as diet, to curb the abdominal obesity epidemic and associated disease risks in China.

Consumption of red meat (RM), specifically processed $\mathrm{RM}$, has been associated with greater WC gain and an increased risk of abdominal obesity in Western populations, although the evidence is inconsistent. Some studies have suggested a positive association between consumption of RM and processed RM and WC gain and abdominal obesity ${ }^{10,11}$ or have found a null association, ${ }^{12-14}$ and other studies indicate a beneficial role for RM consumption among adults. ${ }^{15} \mathrm{RM}$ consumption patterns vary across countries. Unlike Western populations, Chinese adults have a relatively low intake of total RM, including a much lower proportion of processed RM, and pork has been the predominant type of fresh RM consumed (over 90\%). ${ }^{16}$ Given the differences in types and quantities of individual RM consumption, the associations of RM consumption with WC and abdominal obesity in the Chinese population may be different from those in Western populations. To date the possible association between RM consumption and abdominal obesity in the Chinese population has not been examined.

The currently accepted recommendation is to consume moderate amounts of lean fresh RM. ${ }^{17,}{ }^{18}$ However, studies to determine the potentially different health influences of fatty versus lean RM are limited. In China RM is distinguished as either lean or fatty RM (with excessive fat retained in all fatty RM), providing a unique opportunity to study this dimension of the RM intake of a population over time. ${ }^{19}$

The present study investigated the association between intakes of RM and its subtypes (fatty versus lean fresh RM) and the risk of increased WC and abdominal obesity in Chinese adults from the China Health and Nutrition Survey (CHNS), an ongoing, large-scale, longitudinal, prospective cohort survey (1993-2011). 


\section{METHODS}

\section{Study Population}

All data used in this study were derived from the CHNS. The CHNS was initiated in 1989 and has been followed up every two to four years with a focus on assessing the relationships between the economic, sociological, and demographic transformation in China and the resulting effects on the health and nutritional status of the Chinese population. The CHNS used a multistage, random cluster process to draw the sample from the original eight provinces, and communities were selected randomly as the primary sampling units. The sampling procedure has been described in detail elsewhere. ${ }^{20}$ Such sampling reflects the hierarchical data structure of the CHNS: measurement occasions (level 1) for individuals (level 2) nested in communities (level 3).

Our analysis used the seven waves of survey data between 1993 and 2011, because the WC measurement was added to the CHNS in 1993. Of all the participants aged 18 to 75 who had complete data on dietary, anthropometric, demographic, socioeconomic, and other lifestyle factors in a survey year, we excluded pregnant or lactating women, those having implausible energy intakes $(<800$ kilocalories [kcal] per day or $>6,000 \mathrm{kcal}$ for men and $<600 \mathrm{kcal}$ or $>$ $4,000 \mathrm{kcal}$ for women $),{ }^{21}$ and those having unrealistic WCs $(<50$ centimeters $[\mathrm{cm}]$ or $>130$ $\mathrm{cm})$. The current analysis therefore consists of 16,822 participants (8,089 males; 8,733 females) clustered in 236 communities, resulting in 47,785 total responses in the seven survey years.

The protocol of the survey was approved by the institutional review committees of the University of North Carolina at Chapel Hill and the National Institute of Nutrition and Food Safety, Chinese Center for Disease Control and Prevention. All subjects gave written informed consent for their participation in the survey.

\section{Dietary Assessment}

We assessed dietary intake at the individual level in three consecutive 24-hour dietary recalls (two weekdays and one weekend day) in each wave of the CHNS. Trained health workers interviewed the participants on each of those days to collect the types and amounts of all food and beverage items (measured in grams [g]) consumed during the preceding 24 hours. ${ }^{20}$

We collected information on home cooking oil and condiment consumption of all the family members with a household food inventory weighing method on the same three days as the 24-hour recall. We determined the percentage of the oil and condiments from the home inventory that each member consumed by the ratio of his or her energy intake to the energy intake of all family members. We calculated the individual total energy intake (TEI) by linking dietary intake data to the China Food Composition Table. ${ }^{22}$ We used the average intake of RM, other foods and TEI for each individual from the three consecutive 24-hour recalls. 


\section{Red Meat Groups}

We classified RM as fresh RM and processed RM. Fresh RM included all muscle and organ meat from pork, beef, and mutton that had not been treated, whereas processed RM included the products of all types of RM that had undergone treatment, such as sausages, salami, ham, and luncheon meats. We further divided fresh RM into fatty fresh RM ( $\geq 10 \mathrm{~g}$ fat/100 g of edible fresh RM) and lean fresh RM $(<10 \mathrm{~g}$ fat $/ 100 \mathrm{~g}$ of edible fresh RM) based on the food grouping system developed by Barry M. Popkin et al. ${ }^{20}$

Given the considerable proportion of participants who did not consume RM or certain types of RM during the three-day survey period, our analysis categorizes the intakes of RM and each subtype into five levels separately by gender to reflect nonconsumers and quartiles of intake among corresponding consumers. The lowest quartile of intake of RM or a subtype is the reference group.

\section{Definition of Abdominal Obesity and Overweight-Obesity Status}

At each visit trained health workers measured individual WCs midway between the lowest rib and the iliac crest with a SECA tape measure. Adult abdominal obesity is defined as a WC of $\geq 85 \mathrm{~cm}$ for men and $\geq 80 \mathrm{~cm}$ for women according to the guidelines of the Working Groups for Obesity in China. ${ }^{23}$ We used the Chinese body mass index (BMI) cutoff of 24 kilograms per square meter $\left(\mathrm{kg} / \mathrm{m}^{2}\right)$ to determine overweight. ${ }^{23}$ Too few Chinese have a BMI of 28 or higher to permit a second obesity cutoff.

\section{Assessment of Other Covariates}

Trained interviewers used questionnaires to collect information on annual family income, individual education level, physical activity, smoking status, and alcohol consumption. We calculated per capita annual family income by dividing annual family income by household size. We inflated the per capita annual family incomes in each survey to 2011 values by adjusting for the consumer price index and then divided them into tertiles (low, medium, and high). We measured education level by the number of years of formal education completed in regular school.

The community urbanicity index, a complex measure of urbanization, is based on 12 multidimensional components reflecting economic, social, demographic, and infrastructural diversity at the community level. ${ }^{20}$ This variable is a continuous time-varying index, and higher values indicate a higher degree of urbanicity.

Physical activity includes four domains: occupational, household chore, leisure time, and transportation activities. All activities were reported in average hours per week during the past year. ${ }^{3}$ We converted time spent in each activity into metabolic equivalent of task (MET) hours per week based on the Compendium of Physical Activities. We categorized the sums of total physical activity into tertiles (light, moderate, and heavy).

The other covariates we considered as potential confounders in the models include age (in years); smoking status (dichotomized as $1=$ current smoker and $0=$ former or never smoker); alcohol consumption (dichotomized as $1=$ yes and $0=$ no); chronic disease (dichotomized as $1=$ yes if diagnosed by a doctor as hypertension, diabetes, stroke, or 
myocardial infarction and $0=$ no); and other relevant dietary intake variables, including total energy (used for energy adjustment), grains and cereals, vegetables and fruits, soybeans and nuts, cooking oil, and other animal foods. ${ }^{13}, 24,25$

\section{Statistical Analyses}

We categorized participants into nonconsumers of RM and quartiles of RM intake at baseline. We used chi-square tests (categorical variables) and one-way analysis of variance (ANOVA) or analysis of covariance (ANCOVA) (continuous variables) to test differences in the general characteristics of participants across the RM intake levels at baseline.

We constructed three-level mixed-effects linear regression models to estimate WC in relation to RM intake and performed three-level mixed-effect logistic regression models to assess the risk of abdominal obesity. We calculated regression coefficients (95\% confidence intervals [CIs]) and odds ratios (ORs) (95\% CIs), respectively. We conducted all analyses separately for the intakes of RM and its subtypes and stratified them by gender. We constructed three sequential models. Model 1 includes age only. Model 2 is additionally adjusted for income, education, urbanicity index, physical activity, smoking status, alcohol consumption, and chronic disease history. Model 3 is further adjusted for total energy intake and other potential dietary confounders. Moreover, we evaluated linear trends by assigning participants the median value for quartiles of intake of RM and its subtypes, and we entered this variable as a continuous term in the regression model. We used likelihood ratio tests to examine the potential confounders, effect modifier, and goodness of fit between the models. The current analysis dichotomized processed RM intake to represent whether or not participants consumed it and considered it as one confounder in the models.

All statistical tests were two-tailed, and we regarded differences as significant at $\mathrm{p}<0.05$. For all analyses we used SAS (version 9.2, SAS Institute Inc., NC) and Stata/SE (Intercooled STATA, version 12.0, StataCorp, TX).

\section{RESULTS}

The selected characteristics of participants across RM intake levels at baseline are summarized in table 1 . Men tended to have a slightly higher median intake of RM than did women ( $\mathrm{p}<0.001$ ). Increasing total RM intake was positively related to a higher proportion of smoking and alcohol consumption and higher BMIs and WCs only in men. Both men and women with higher RM intakes tended to live in highly urbanized communities and have higher socioeconomic statuses, lower physical activity levels, and greater intakes of other types of animal foods and cooking oil. It is notable that both men and women who did not consume RM had the highest intakes of grains and cereals and vegetables and fruits and the lowest intakes of cooking oil and other meat across the total RM intake levels.

Table 2 shows the longitudinal association between levels of RM and subtype consumption and WC stratified by gender. Men showed significant WC increases of $0.74 \mathrm{~cm}(95 \% \mathrm{CI}$ : 0.39-1.09) from a higher intake of total fresh RM and $0.59 \mathrm{~cm}$ (95\% CI: 0.38-1.09) from a higher intake of fatty fresh RM but not from a higher intake of lean fresh RM in the top versus the bottom quartile when adjusted for all potential confounders. In women we 
observed no significant association of consumption of RM or its subtypes with WC. With regard to nonconsumers of RM or its subtypes, WC was not significantly lower in either men or women compared with consumers in the lowest quartile of intake.

As table 3 shows, the observed positive association in men persisted but attenuated after additional adjustment for baseline WC. Comparing the highest with the lowest quartiles of intake of total RM, fresh RM, and fatty fresh RM, ORs (95\% CI) of abdominal obesity were 1.27 (1.07-1.49), 1.25 (1.06-1.47), and 1.22 (1.03-1.44), respectively. In women we found a similar positive association with risk of abdominal obesity in the comparison of the highest and the lowest quartiles of intake of fresh RM (OR: 1.16; 95\% CI: 1.01-1.34) and fatty fresh RM (OR: 1.17; 95\% CI: 1.01-1.36). We observed no significant association between intake of lean fresh RM and risk of abdominal obesity in either men or women.

In stratified analyses by BMI categories, we observed significant positive associations of fatty fresh RM consumption with WC gain $(0.79 \mathrm{~cm}$; $95 \%$ CI: $0.39-1.20)$ and abdominal obesity risk (OR: 1.29; 95\% CI: 1.04-1.61) in men in the normal weight category but not among overweight or obese men. In addition we found a significant positive association of fatty fresh RM intake with WC gain and abdominal obesity risk only among male drinkers (data not shown).

\section{DISCUSSION}

In this population-based, prospective cohort study of the Chinese population, we found that intake of total RM, intake of fresh RM, and intake of fatty fresh RM were positively associated with an increased WC measurement (men only) and risk of abdominal obesity among Chinese adults aged 18 to 75 . However, we observed no significant associations with lean fresh RM intake in either men or women. To the best of our knowledge, the present study is the first that investigates the longitudinal association of RM intake with WC and abdominal obesity in Chinese adults with an emphasis on the potentially different roles of fatty and lean fresh RM.

Previous studies have reported inconsistent results, including positive, ${ }^{26}$ null, ${ }^{12-14}$ or negative associations between RM consumption and WC gain. ${ }^{15}$ However, J. Halkjaer et al. found that the positive association of RM consumption on WC gain seemed to shift to no association or an adverse association at high intakes. ${ }^{15}$ Moreover, Y. Wang and M. A. Beydoun found a cross-sectional positive association between RM intake and abdominal obesity among US adults. ${ }^{11}$ Another prospective study suggested an 8.1 times greater risk of central obesity incidence among 717 Spanish adults with extreme baseline RM intakes. ${ }^{10}$ That study, which had a small sample size and only a one-year follow-up, included older participants with high cardiovascular risk and indicated mixed effects of unprocessed and processed RM intakes. Of note, the lack of standard definitions of RM, WC sites, and abdominal obesity make comparisons across studies difficult. ${ }^{18}$ Generally speaking, our findings of a positive association between RM intake and abdominal obesity in Chinese men and women are consistent with those of studies using similar RM definitions. ${ }^{11,15}$ 
Studies of the potentially different association of fatty versus lean fresh RM with WC and abdominal obesity are lacking. Our study defined lean fresh RM as less than $10 \mathrm{~g}$ of fat per $100 \mathrm{~g}$ of raw pork, beef, or lamb. Our findings suggest negative association with WC and abdominal obesity only from intake of fatty fresh RM, which may be due to its higher content of saturated fats, heme iron, protein, and energy compared with lean fresh RM. ${ }^{17,18,27,28}$

The gender-specific associations with WC observed in our study are possibly due to the differences in physiological status and lifestyle, such as alcohol consumption and tobacco smoking. Smoking and alcohol consumption rates were much lower among women than among men and were not different across RM intake levels. Our study also found that male drinkers had higher WCs. In addition women had slightly lower intakes of RM and lower WCs, so the variation might not be large enough to detect an association. Gender-specific biological mechanisms are not understood well and warrant further study.

Our study has many strengths, including its large participant size with a wide age range, prospective cohort design with clear temporality, and adjustment for a comprehensive range of potential confounders. Most importantly, we examined gender-specific consumption of fatty versus lean fresh RM and found a null association of lean fresh RM with abdominal obesity risk among Chinese men and women. This finding may lend some support to the essential role of a moderate intake of lean fresh RM, ${ }^{17,} 18$ especially in the context of the double burden of nutrient deficiency and obesity-related chronic diseases in developing countries, including China. In addition interviewer-administered 24-hour dietary recalls can capture extensive and complete information on all foods and beverages consumed and provide a more precise assessment of intake of RM and its subtypes than self-reported food frequency methods. Further, mixed-effect modeling contributed to less biased, more precise estimates.

Our study also had several limitations. First, three consecutive 24-hour dietary recalls may have relatively limited correction for within-subject variation compared to nonconsecutive 24-hour recalls. However, the average intake over three days can offer a relatively valid estimate of usual RM intakes, ${ }^{25}$ as was shown in earlier research using the CHNS. ${ }^{29}$ As well, the diet data collected between August and November of each wave may not reflect seasonal differences in RM consumption. Second, our analysis did not consider RM cooking methods that might modify or confound the association of RM intake with certain health outcomes, particularly as the proportion of dishes stir-fried and deep-fried has increased remarkably in the past two decades. ${ }^{30}$ Further, we did not examine the health effects of long-term RM intake, which is another avenue for future research with the CHNS.

In conclusion, the present study provides some evidence of a positive association between the intakes of total RM and fatty fresh RM and abdominal obesity in Chinese adults aged 18 to 75. Our findings lend support to the recommendation of a moderate intake of lean fresh RM. At the same time, the gender-specific differential associations of fatty versus lean fresh RM warrant further investigation. 


\section{Acknowledgments}

\section{Funding}

This study was funded by the Institute of Nutrition and Food Safety, Chinese Center for Disease Control and Prevention, China; the National Institutes of Health (NIH), USA (R01-HD30880 and R01-HD38700); and the Fogarty International Center, NIH, USA.

\section{References}

1. Popkin BM, Adair LS, Ng SW. Global nutrition transition and the pandemic of obesity in developing countries. Nutr Rev. 2012; 70:3-21. [PubMed: 22221213]

2. Fengying Z, Du Shufa, Wang Zhihong, Zhang J, Du Wenwen, Popkin Barry M. Dynamics of the Chinese Diet and the Role of Urbanization, 1991-2011. Obes Rev. 2014; 15 (Suppl 1):16-26. [PubMed: 24341755]

3. Ng SW, Howard Annie-Green, Wang Huijun, Su Chang, Zhang Bing. The Physical Activity Transition among adults in China: 1991-2011. Obes Rev. 2014; 15 (Suppl 1):27-36. [PubMed: 24341756]

4. Yan S, Li J, Li S, Zhang B, Du S, Gordon-Larsen P, Adair L, Popkin B. The expanding burden of cardiometabolic risk in China: the China Health and Nutrition Survey. Obes Rev. 2012; 13:810821. [PubMed: 22738663]

5. Adair LS, Gordon-Larsen Penny, Du Shufa, Zhang Bing, Popkin Barry. The emergence of cardiometabolic disease risk in in Chinese children and adults: consequences of changes patterns of diet, physical activity, and obesity. Obes Rev. 2014; 15 (Suppl 1):49-59. [PubMed: 24341758]

6. Yang ZJ, Liu J, Ge JP, Chen L, Zhao ZG, Yang WY. Prevalence of cardiovascular disease risk factor in the Chinese population: the 2007-2008 China National Diabetes and Metabolic Disorders Study. Eur Heart J. 2012; 33:213-220. [PubMed: 21719451]

7. Gordon-Larsen P, Adair LS, Meigs JB, Mayer-Davis E, Herring A, Yan SK, Zhang B, Du S, Popkin BM. Discordant risk: overweight and cardiometabolic risk in Chinese adults. Obesity (Silver Spring). 2013; 21:E166-174. [PubMed: 23505200]

8. Janssen I, Katzmarzyk PT, Ross R. Waist circumference and not body mass index explains obesityrelated health risk. Am J Clin Nutr. 2004; 79:379-384. [PubMed: 14985210]

9. Wang HJ, Wang ZH, Yu WT, Zhang B, Zhai FY. Changes of waist circumference distribution and the prevalence of abdominal adiposity among Chinese adults from 1993 to 2006. Chinese Journal of Epidemiology. 2008; 29:953-958. [PubMed: 19173871]

10. Babio N, Sorli M, Bullo M, Basora J, Ibarrola-Jurado N, Fernandez-Ballart J, Martinez-Gonzalez MA, Serra-Majem L, Gonzalez-Perez R, Salas-Salvado J, Nureta PI. Association between red meat consumption and metabolic syndrome in a Mediterranean population at high cardiovascular risk: cross-sectional and 1-year follow-up assessment. Nutr Metab Cardiovasc Dis. 2012; 22:200207. [PubMed: 20875949]

11. Wang Y, Beydoun MA. Meat consumption is associated with obesity and central obesity among US adults. Int J Obes (Lond). 2009; 33:621-628. [PubMed: 19308071]

12. Nooyens AC, Visscher TL, Schuit AJ, van Rossum CT, Verschuren WM, van Mechelen W, Seidell JC. Effects of retirement on lifestyle in relation to changes in weight and waist circumference in Dutch men: a prospective study. Public Health Nutr. 2005; 8:1266-1274. [PubMed: 16372922]

13. Drapeau V, Despres JP, Bouchard C, Allard L, Fournier G, Leblanc C, Tremblay A. Modifications in food-group consumption are related to long-term body-weight changes. Am J Clin Nutr. 2004; 80:29-37. [PubMed: 15213024]

14. Halkjaer J, Sorensen TI, Tjonneland A, Togo P, Holst C, Heitmann BL. Food and drinking patterns as predictors of 6-year BMI-adjusted changes in waist circumference. Br J Nutr. 2004; 92:735748. [PubMed: 15522143]

15. Halkjaer J, Tjonneland A, Overvad K, Sorensen TI. Dietary predictors of 5-year changes in waist circumference. J Am Diet Assoc. 2009; 109:1356-1366. [PubMed: 19631041] 
16. Lee JE, McLerran DF, Rolland B, Chen Y, Grant EJ, Vedanthan R, Inoue M, Tsugane S, Gao YT, Tsuji I, et al. Meat intake and cause-specific mortality: a pooled analysis of Asian prospective cohort studies. Am J Clin Nutr. 2013; 98:1032-1041. [PubMed: 23902788]

17. Popkin BM. Reducing meat consumption has multiple benefits for the world's health. Archives of internal medicine. 2009; 169:543-545. [PubMed: 19307515]

18. McAfee AJ, McSorley EM, Cuskelly GJ, Moss BW, Wallace JM, Bonham MP, Fearon AM. Red meat consumption: an overview of the risks and benefits. Meat Sci. 2010; 84:1-13. [PubMed: 20374748]

19. Wang Z, Tang L, Sun G, Tang Y, Xie Y, Wang S, Hu X, Gao W, Cox SB, Wang JS. Etiological study of esophageal squamous cell carcinoma in an endemic region: a population-based case control study in Huaian, China. BMC cancer. 2006; 6:287. [PubMed: 17173682]

20. Jones-Smith JC, Popkin BM. Understanding community context and adult health changes in China: development of an urbanicity scale. Soc Sci Med. 2010; 71:1436-1446. [PubMed: 20810197]

21. Wang Z, Zhang B, Wang H, Zhang J, Du W, Su C, Zhang J, Zhai F. Study on the multilevel and longitudinal association betweend meat consumption and changes in body mass index, body weight and risk of incident overweight among Chinese adults. Chin J Epidemiol. 2013; 34:1-7.

22. Yang, Y.; Wang, G.; Pan, X. China Food Composition Table 2009. 2. Beijing: Peking University Medical Press; 2009.

23. Chen C, Lu FC. Department of Disease Control Ministry of Health PRC. The guidelines for prevention and control of overweight and obesity in Chinese adults. Biomed Environ Sci. 2004; 17 (Suppl):1-36. [PubMed: 15807475]

24. Romaguera D, Angquist L, Du H, Jakobsen MU, Forouhi NG, Halkjaer J, Feskens EJ, van der AD, Masala G, Steffen A, et al. Dietary determinants of changes in waist circumference adjusted for body mass index - a proxy measure of visceral adiposity. PLoS One. 2010; 5:e11588. [PubMed: 20644647]

25. Moghaddam E, Vogt JA, Wolever TM. The effects of fat and protein on glycemic responses in nondiabetic humans vary with waist circumference, fasting plasma insulin, and dietary fiber intake. J Nutr. 2006; 136:2506-2511. [PubMed: 16988118]

26. Wagemakers JJ, Prynne CJ, Stephen AM, Wadsworth ME. Consumption of red or processed meat does not predict risk factors for coronary heart disease; results from a cohort of British adults in 1989 and 1999. Eur J Clin Nutr. 2009; 63:303-311. [PubMed: 18000518]

27. Casas-Agustench P, Lopez-Uriarte P, Bullo M, Ros E, Gomez-Flores A, Salas-Salvado J. Acute effects of three high-fat meals with different fat saturations on energy expenditure, substrate oxidation and satiety. Clin Nutr. 2009; 28:39-45. [PubMed: 19010571]

28. Storlien LH, Hulbert AJ, Else PL. Polyunsaturated fatty acids, membrane function and metabolic diseases such as diabetes and obesity. Curr Opin Clin Nutr Metab Care. 1998; 1:559-563. [PubMed: 10565410]

29. Paeratakul S, Popkin BM, Kohlmeier L, Hertz-Picciotto I, Guo X, Edwards LJ. Measurement error in dietary data: implications for the epidemiologic study of the diet-disease relationship. Eur J Clin Nutr. 1998; 52:722-727. [PubMed: 9805218]

30. Wang Z, Zhai F, Du S, Popkin B. Dynamic shifts in Chinese eating behaviors. Asia Pac J Clin Nutr. 2008; 17:123-130. [PubMed: 18364337] 


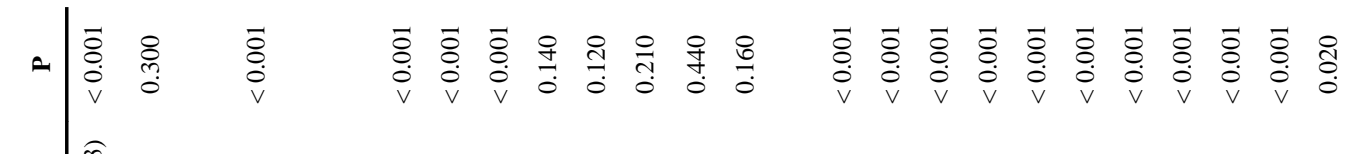

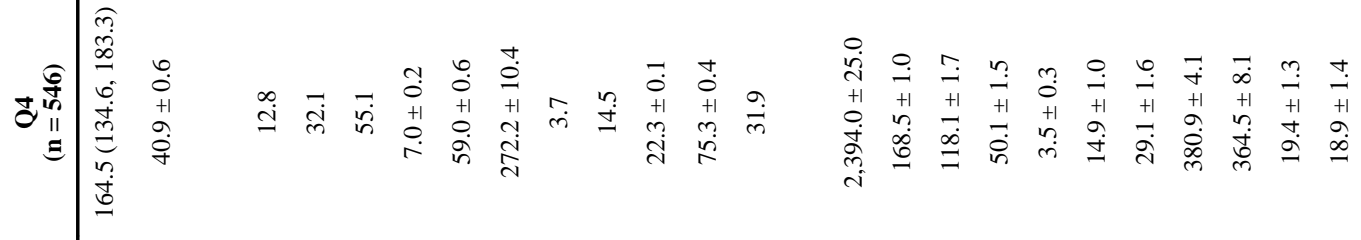

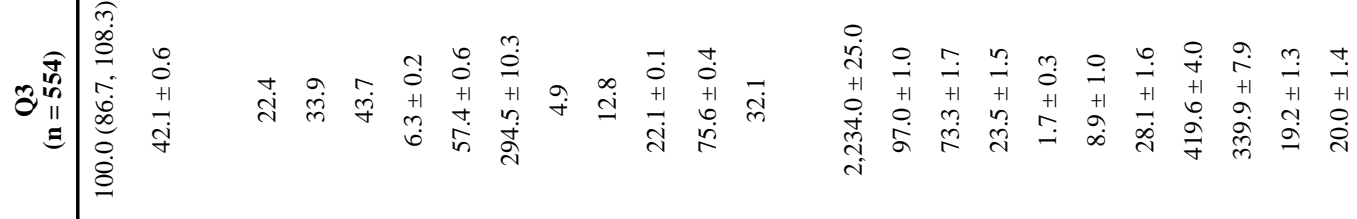

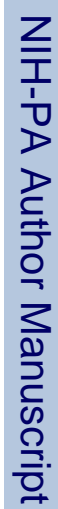

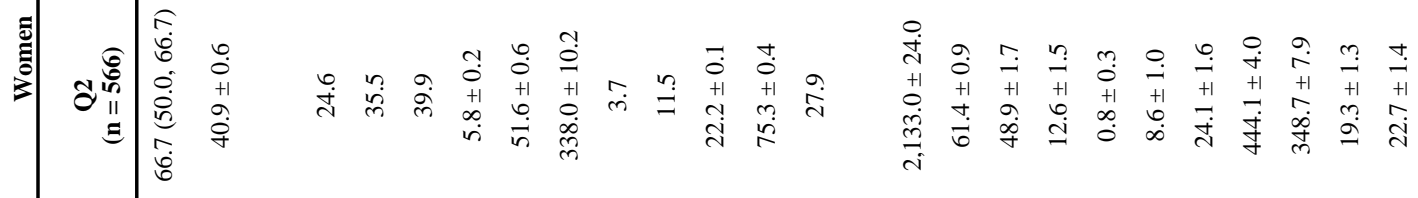

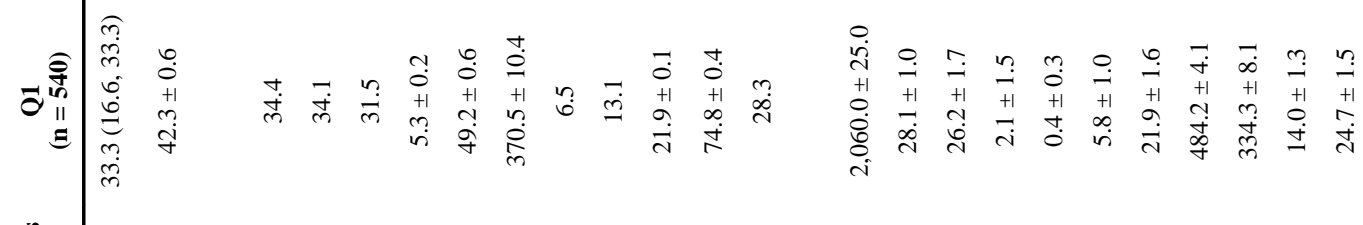

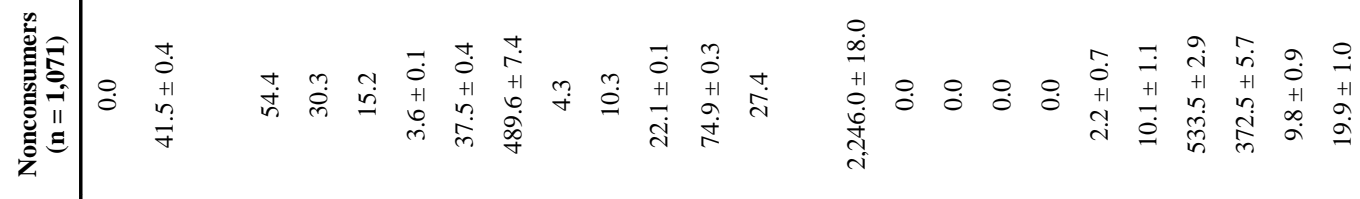

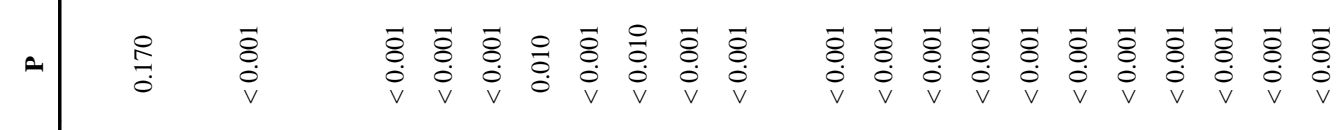

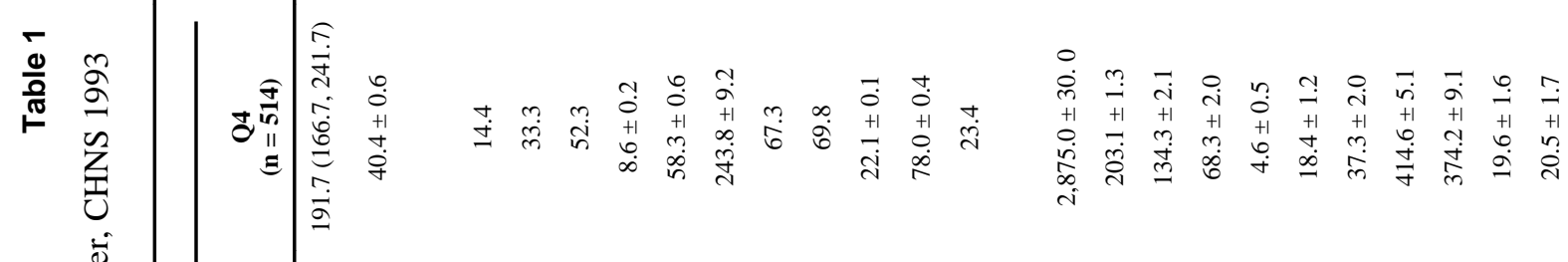

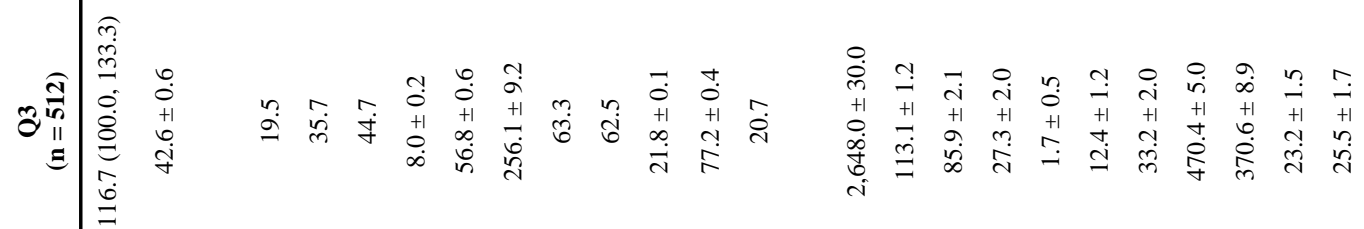

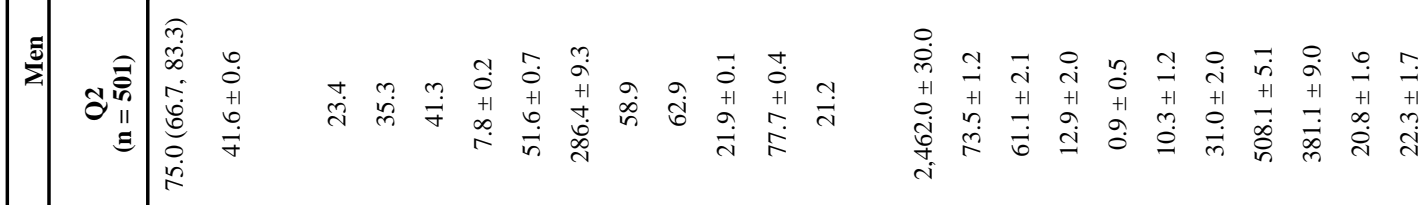

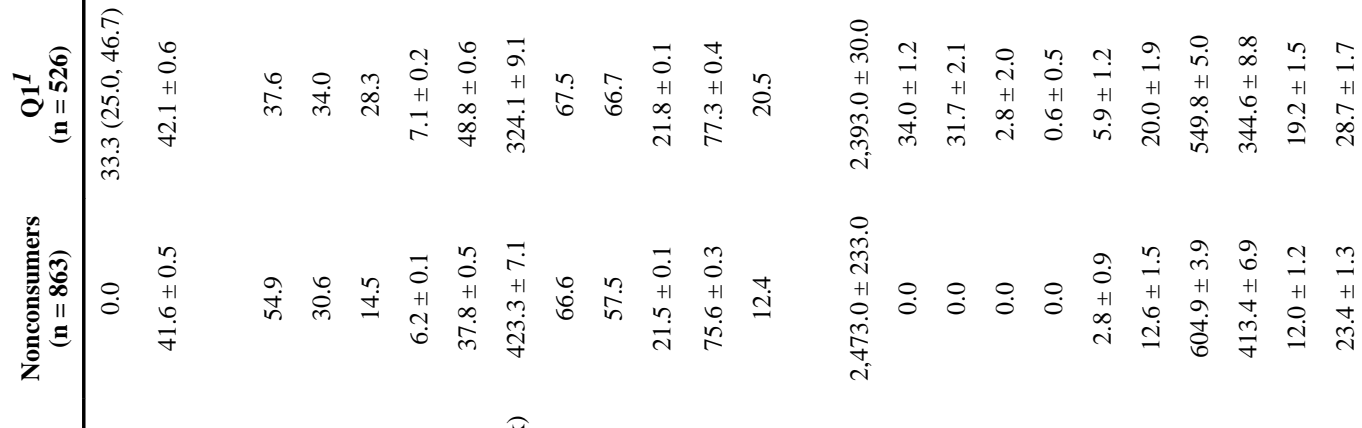

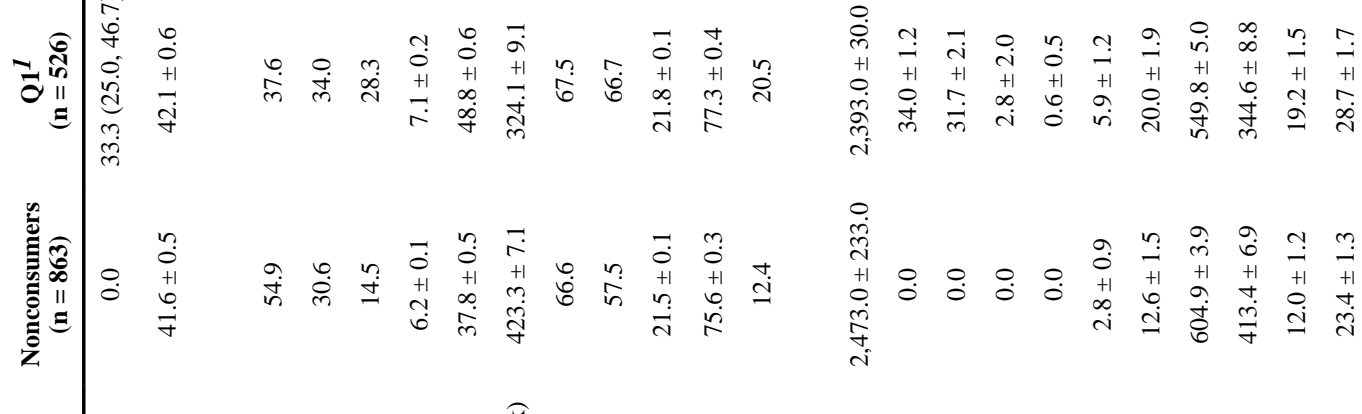




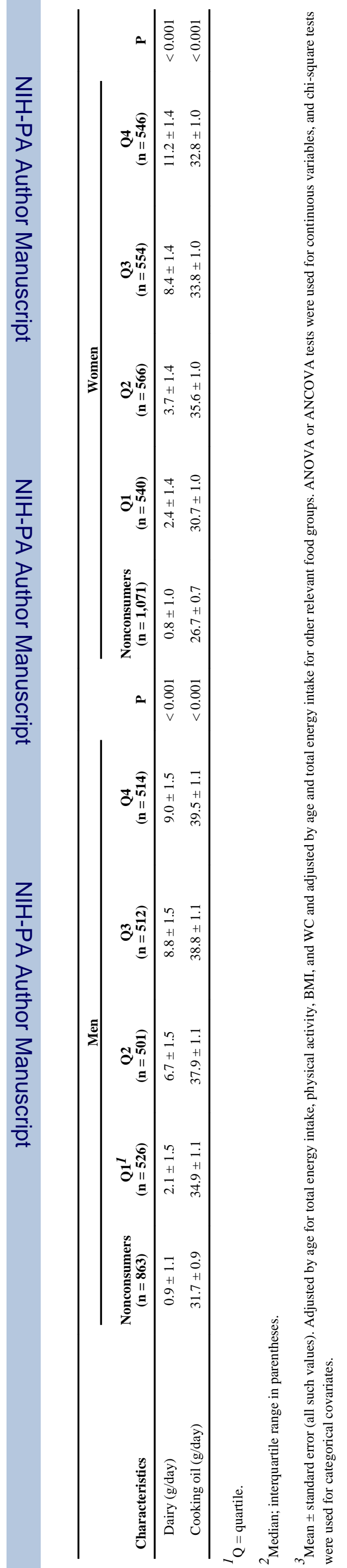




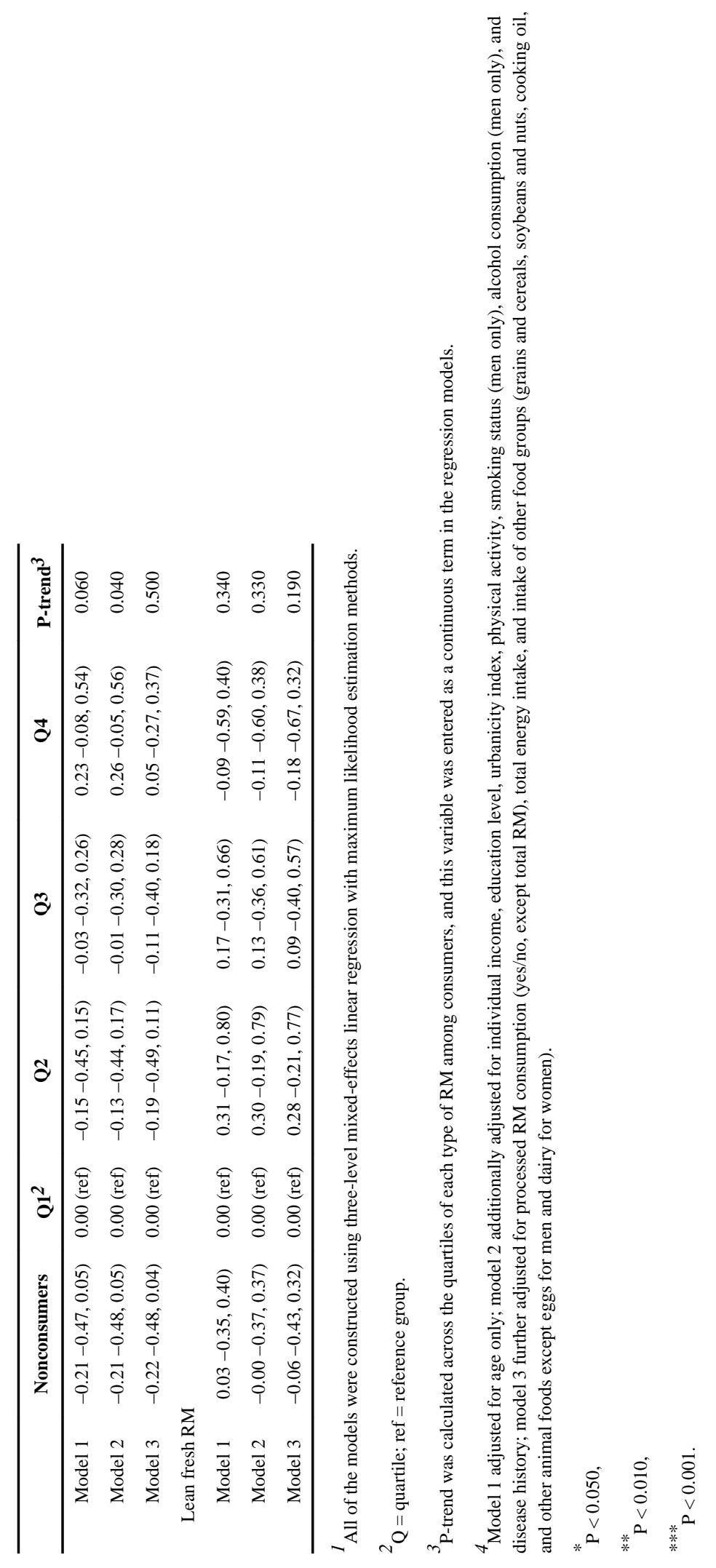




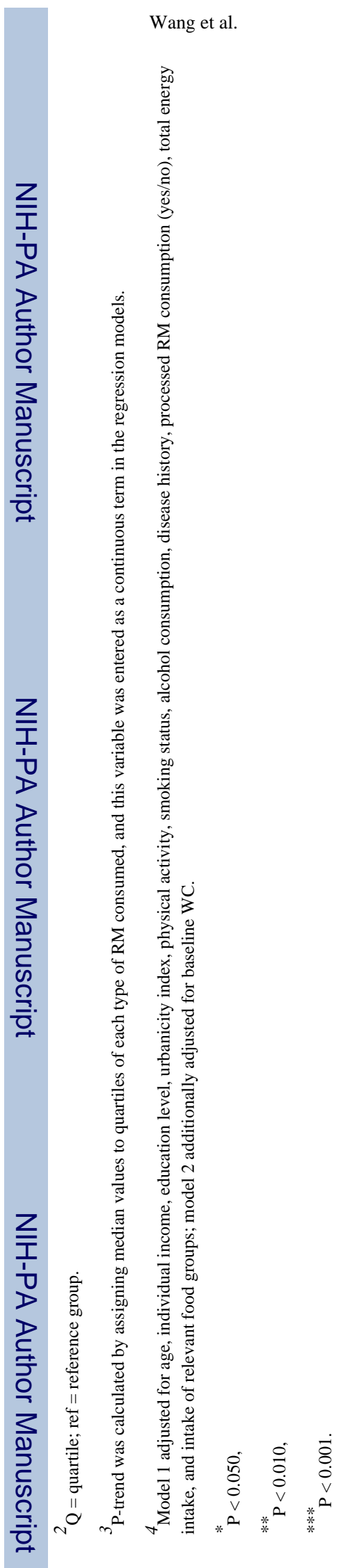

Page 15

Nutr Metab Cardiovasc Dis. Author manuscript; available in PMC 2015 August 01. 